\title{
Perifollicular Fibrosis: Pathogenetic Role in Androgenetic Alopecia
}

\author{
Hyeon Gyeong Yoo, Jin Sook Kim, Se Rah Lee, Hyun Keol Pyo, Hyung In Moon, \\ Jong Hee Lee, Oh Sang Kwon, Jin Ho Chung, Kyu Han Kim, Hee Chul Eun, and \\ Kwang Hyun $\mathrm{CHO}^{*}$
}

Department of Dermatology, Seoul National University College of Medicine, Laboratory of Cutaneous Aging and Hair Research, Clinical Research Institute, Seoul National University Hospital, and Institute of Dermatological Science, Seoul National University; Seoul, 110-744, Korea.

Received October 12, 2005; accepted December 13, 2005

\begin{abstract}
Androgenetic alopecia (AGA) is a dihydrotestosterone (DHT)-mediated process, characterized by continuous miniaturization of androgen reactive hair follicles and accompanied by perifollicular fibrosis of follicular units in histological examination. Testosterone $\left(\mathrm{T}: 10^{-9}-10^{-7} \mathrm{M}\right)$ treatment increased the expression of type $I$ procollagen at mRNA and protein level. Pretreatment of finasteride $\left(10^{-8} \mathrm{M}\right)$ inhibited the T-induced type I procollagen expression at mRNA $(\mathbf{4 0 . 2 \%})$ and protein levels $(24.9 \%)$. $T$ treatment increased the expression of transforming growth factor-beta 1 (TGF- $\beta 1$ ) at protein levels by $81.9 \%$ in the human scalp dermal fibroblasts (DFs). Pretreatment of finasteride decreased the expression of TGF- $\beta 1$ protein induced by an average of T (30.4\%). The type I procollagen expression after pretreatment of neutralizing TGF- $\beta 1$ antibody $(10 \mu \mathrm{g} / \mathrm{ml}) \mathrm{was}$ inhibited by an average of $54.3 \%$. Our findings suggest that T-induced TGF- $\beta 1$ and type I procollagen expression may contribute to the development of perifollicular fibrosis in the AGA, and the inhibitory effects on T-induced procollagen and TGF- $\beta 1$ expression may explain another possible mechanism how finasteride works in AGA.
\end{abstract}

Key words fibrosis; finasteride; androgenetic alopecia; type I procollagen; TGF- $\beta 1$

Androgenetic alopecia (AGA) is undoubtedly the most common form of hair loss in males and androgen, especially dihydrotestosterone (DHT) is universally accepted as a main culprit. ${ }^{1)}$ Recently, the pathogenetic role of type II $5 \alpha$ reductase $(5 \alpha-\mathrm{R})$ transforming testosterone $(\mathrm{T})$ into dihydrotestosterone (DHT) in AGA has been discovered. ${ }^{1,2)}$ For that reason, finasteride, an inhibitor of selective type II $5 \alpha$-R was introduced as a drug and has been widely used as a promising treatment for male pattern baldness, because it decreases DHT levels both in serum and scalp. ${ }^{3,4)}$ Nonetheless, the exact underlying mechanisms how DHT plays a role in AGA is not well understood. ${ }^{1-4)}$

On close histological observation of balded scalp biopsies, the miniaturization of terminal hairs is a distinguishing feature in AGA and is frequently associated with perifollicular fibrosis. ${ }^{5,6)}$ The miniaturization of the hair follicles was found to be associated with a deposit of so-called "collagen or connective tissue streamers" beneath the follicle ${ }^{7)}$ as well as a $2-2.5$ times enlargement of the follicular dermal sheath composed of densely packed collagen bundles. ${ }^{5)}$ Transforming growth factor-beta 1 (TGF- $\beta 1$ ) has been proposed to play an important role in catagen regulation. TGF- $\beta 1$ has a negative growth-regulatory effect on hair follicles in vitro. ${ }^{8)}$

The aim of this study was to find out the mechanisms how androgen causes AGA in the aspect of perifollicular fibrosis by observing the effect of $\mathrm{T}$ and finasteride on the expression of type I procollagen and TGF- $\beta 1$ in cultured human scalp follicular DFs.

\section{MATERIALS AND METHODS}

Antibodies and Chemicals Anti- $\alpha 1(\mathrm{I})$ procollagen amino-terminal extension peptide (SP1.D8) monoclonal antibody (Developmental Studies Hybridoma Bank, Iowa City, MA, U.S.A.) was used as primary antibody at $1: 2000$ for western blotting. T $\left(10^{-9}-10^{-7} \mathrm{M}\right.$; Fluka, Buchs SG,
Switzerland), finasteride $\left(10^{-7} \mathrm{M}\right.$; Sigma-Aldrich, Bornem, Belgium) were used after dissolution either in dimethyl sulfoxide (DMSO) or absolute ethanol (Sigma, St. Louis, MO, U.S.A.) on human scalp cell culture.

Culture of Scalp DFs Skin biopsy specimens were obtained from the scalp of healthy volunteers (average mid 20 years of age) who had not received any medication for at least 1 month. Primary human scalp DFs was isolated from hair follicle interface of mid-dermis portion. After the cultured 3 or 4 passage cells were used in these study. Outgrown cells from explants were cultured in Dulbecco's modified Eagle's media (DMEM) supplemented with glutamine ( $2 \mathrm{~mm})$, penicillin (400 $\mathrm{U}$ per $\mathrm{ml})$, streptomycin $(50 \mathrm{mg} / \mathrm{ml})$, and $10 \%$ FBS in a humidified $5 \% \mathrm{CO}_{2}$ atmosphere at $37{ }^{\circ} \mathrm{C}$. This study was approved by the Institutional Review Board at the Seoul National University Hospital, and all subjects gave the written informed consent.

RT-PCR Total RNA $(1 \mu \mathrm{g})$ from each sample was reverse-transcribed using $2.5 \mu \mathrm{mol} / 1$ random hexamer, $5 \mu \mathrm{mol} / 1 \mathrm{MgCl}_{2}, 50 \mu \mathrm{mol} / 1 \mathrm{KCl}, 10 \mu \mathrm{mol} / 1$ Tris- $\mathrm{HCl}[\mathrm{pH}$ 8.3], $1 \mu \mathrm{mol} / 1$ each of deoxy-nucleotide triphosphate (dNTP), $1.0 \mathrm{U} / \mu \mathrm{l}$ RNase inhibitor (Takara, Tokyo, Japan), and 2.5 $\mathrm{U} / \mu 1 \mathrm{l}$-MLV reverse transcriptase (Gibco-BRL, Gaitherberg, MD, U.S.A.). The following primers were used for detecting $\alpha 1$ (I) procollagen-: sense primer, 5'-CTC GAG GTG GAC ACC ACC CT-3'; antisense primer, 5'-CAG CTG GAT GGC CAC ATC GG-3'. As an internal control, glyceraldehyde-3-phosphate dehydrogenase $(\mathrm{G} 3 \mathrm{PDH})$ was performed. The products were analyzed by $2 \%$ agarose gel electrophoresis.

Western Blot DFs obtained from frontal scalp hair follicles were grown up on the $6 \mathrm{~cm}$ polystyrene dishes. Briefly, confluent DFs were cultured for $24 \mathrm{~h}$ in serum-free DMEM, and then treated for $1 \mathrm{~d}$ with either $10^{-9}-10^{-7} \mathrm{M}$ of $\mathrm{T}$ or $\mathrm{fi}-$ nasteride $\left(10^{-8} \mathrm{M}\right)$. To elucidate the effect of neutralizing TGF- $\beta 1$ antibody on the procollagen expression, we are pre- 
treated with the dose of antibody before $\mathrm{T}$ treatment in DFs. To determine the amounts of type I procollagen protein secreted into culture media, equal aliquots of conditioned culture media from an equal number of cells were fractionated by $10 \%$ SDS-PAGE, transferred to Hybond ECL membrane (Amersham Biosciences, Buckinghamshire, England). The membrane was soaked overnight in 5\% skim milk in PBS $/ 0.05 \%$ Tween 20 at $4{ }^{\circ} \mathrm{C}$ and then incubated with antiSP1.D8 monoclonal antibody of a $1: 2000$ dilution in 5\% skim milk in PBS $/ 0.05 \%$ Tween 20 at $4{ }^{\circ} \mathrm{C}$ overnight. After being washed three times at intervals of $10 \mathrm{~min}$ with PBS $/ 0.05 \%$ Tween 20 , the membranes were incubated with anti-mouse IgG-HRP conjugates $(1: 2000)$ for $1 \mathrm{~h}$ at room temperature and washed twice with TBST. The antibodyantigen complexes were detected using the ECL system (Amersham Pharmacia Biotech; Little Chalfont, U.K.). The results were analyzed using Bio-Rad GS-700 imaging densitometer (Hercules, CA, U.S.A.).

Measurement of TGF- $\boldsymbol{\beta} \boldsymbol{1}$ by ELISA After incubating scalp follicle DFs on the hormone treated dishes for $24 \mathrm{~h}$, we harvested the conditioned medium and then measured the concentration of TGF- $\beta 1$ by ELISA kit (Quantikine human TGF- $\beta 1$ immunoassay, R\&D Systems), according to the manufacturer's instructions.

Statistics Statistical analyses were performed using the Student $t$-test. A $p$-value of less than 0.05 was considered statistically significant.

\section{RESULTS}

Finasteride Inhibited T-Induced Type I Procollagen Expression in Cultured Human Scalp DFs According to the RT-PCR analysis of target gene expression after $\mathrm{T}$ treatment, hair follicular DFs from human scalp expressed type I procollagen mRNA. A semiquantitative study clearly showed the increased expression levels of type I procollagen in follicular DFs in response to T treatment (Fig. 1A), which indicated that this steroid $(\mathrm{T})$ receptors are also present on DFs. When $\mathrm{T}$ was treated into the culture media of DFs, $\mathrm{T}$ was demonstrated to stimulate significantly type I procollagen expression in cultured DFs $(n=6, p<0.05)$. The treatment of T of $10^{-8} \mathrm{M}$ increased significantly type I procollagen production up to approximately $220 \%$ in the culture media of the scalp DFs (Fig. 1B), compared with control (100\%).

In order to investigate whether finasteride $\left(10^{-7} \mathrm{M}\right)$ is involved in T-induced type I procollagen synthesis, finasteride was pretreated to cultured scalp DFs. The $\mathrm{T}\left(10^{-8} \mathrm{M}\right)$ was found to increase the transcription levels of type I $\alpha 1$-procollagen up to $421.1 \%(p<0.01)$, compared with the vehicletreated control $(100 \%)$. Finasteride $\left(10^{-7} \mathrm{M}\right)$ only treatment also increased type I procollagen transcription levels upto $306.7 \%$, compared with the vehicle treated control $(100 \%)$. However, the pre-treatment of finasteride $\left(10^{-7} \mathrm{M}\right)$ before $\mathrm{T}$ $\left(10^{-8} \mathrm{M}\right)$ showed type I collagen gene transcription levels of $251.9 \%$, compared to the vehicle-treated control (100\%), meaning that finasteride inhibited T-induced type I procollagen transcription by $40.2 \%$ (Fig. 1C). These results suggest that finasteride may be associated with androgen (T)-induced mRNA expression of type I $\alpha 1$-procollagen in the human scalp DFs.

Next, we performed western blot analysis for type I pro- collagen protein secreted from the cultured scalp DFs under the same condition above. The levels of type I procollagen protein in T-treated DFs increased significantly upto $260.5 \%$ $(n=5, p<0.01)$. The expression levels of type I procollagen protein in the finasteride/androgen co-treated scalp DFs was significantly lower (FT, 203.5\%, $n=5, p<0.05)$ than those in androgen only treated scalp DFs (Fig. 1D).

Finasteride Inhibited T-Induced TGF- $\beta 1$ Expression Levels To further investigate the molecular mechanisms of androgen-induced type I procollagen expression, we observed the effect of $\mathrm{T}$ on the expression levels of the cytokine, TGF- $\beta 1$, which are known to play an important role in regression phase of mammalian hair cycle. ${ }^{9)}$ The secreted levels of TGF- $\beta 1$ by ELISA were measured in the conditioned media harvested at $24 \mathrm{~h}$ after treating DFs with $\mathrm{T} / \mathrm{fi}-$ nasteride. As shown in Fig. 2, TGF- $\beta 1$ protein levels secreted from DFs treated with $\mathrm{T}$ was increased about 1.82-fold in $24 \mathrm{~h}$ treatment, compared with those of vehicle treated control. Pretreatment of finasteride inhibited the T-induced the expression of TGF- $\beta 1$ protein by an average of $30.4 \%$ $(p<0.05)$.

T Increses Procollagen Expression via TGF- $\beta 1$ To investigate the regulation mechanisms of androgen-induced type I procollagen expression via TGF- $\beta 1$, we observed the effect of $\mathrm{T}$ on the expression levels of type I procollagen by TGF- $\beta 1$ neutralizing antibody. The secreted levels of type I procollagen expression were measured in the conditioned media by treatment of T and TGF- $\beta 1$ antibody. As shown in Fig. 3, type I procollagen level on treatment of $\mathrm{T}\left(10^{-8} \mathrm{M}\right)$ was increased about 3.6-fold in $24 \mathrm{~h}$ treatment, compared with those of vehicle treated control $(p<0.01)$. After the pretreatment of TGF- $\beta 1$ neutralizing antibody $(2,10 \mu \mathrm{g} / \mathrm{ml})$, type I procollagen expression was inhibited by an average of $12.9 \%$ and $54.3 \%(p<0.01)$, respectively.

\section{DISCUSSION}

AGA is hereditary and androgen-dependent, progressive thinning of the scalp hair that follows a defined clinical pattern. Major advances have been achieved in finding out the androgen metabolism involved. Until now, it can be summarized into just two points. One is more peripheral conversion of $\mathrm{T}$ to DHT, a reaction catalyzed by the enzyme $5 \alpha-\mathrm{R}$ in hair follicles from the balded scalp. ${ }^{10)}$ Type II $5 \alpha-\mathrm{R}$ transforms $\mathrm{T}$ into DHT and does so more in the areas of the scalp affected by AGA than in those in which AGA never develops. Compared to T, DHT has approximately five fold greater affinity for the AR and DHT binding to AR in hair follicles is commonly accepted as the first step leading to the miniaturizing of follicles seen in AGA. ${ }^{11-13)}$ The other is higher levels of AR in cells from balding scalp hair follicles with similar properties to those from non-balding scalp. ${ }^{14)}$

The primary location for the AR in hair follicles is well known to be the dermal papillae in both anagen and telogen hairs. ${ }^{15,16)}$ Moreover, it is usually believed that the dermal papilla plays a major regulatory role in hair follicles and is thought to be the site of androgen action. Therefore, action mechanisms of androgen on AGA has been usually studied using dermal papilla cells. ${ }^{14,17-21)}$

Recently, AR has been also identified in the interfollicular DFs and epidermal keratinocytes. ${ }^{15)}$ Therefore, several ef- 
(A)

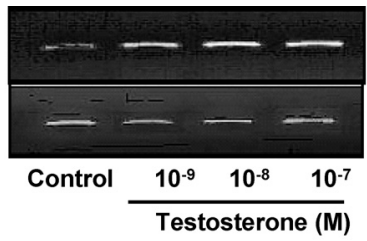

Procollagen $\alpha(\mathrm{l})$

GAPDH

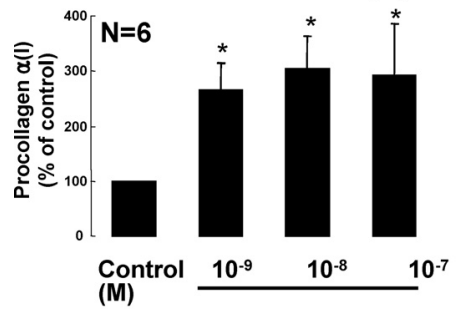

Testosterone

(C)

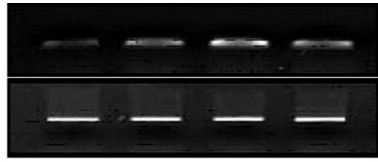

$\begin{array}{llll}C & \mathrm{~F} & \mathrm{~T} & \mathrm{FT}\end{array}$

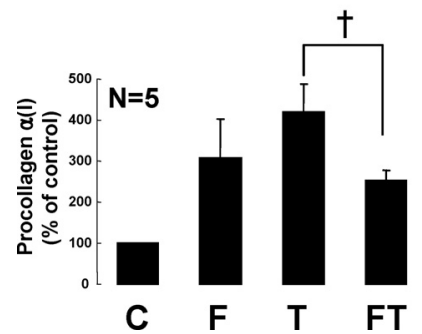

(B)

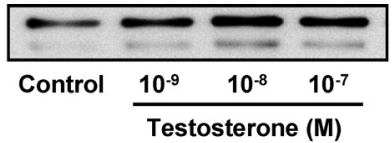

Type I Procollagen

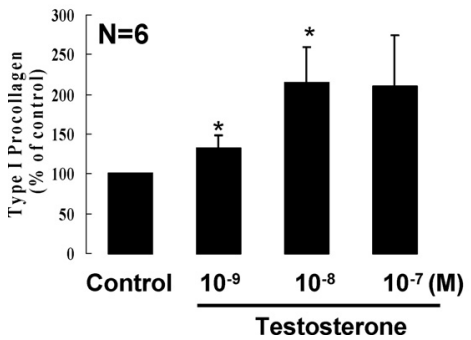

(D)

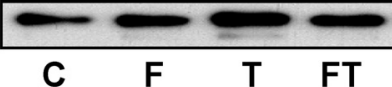

Type I Procollagen

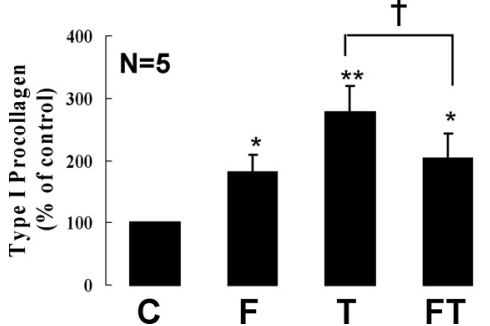

Fig. 1. Expression Level of the Procollagen $\alpha(\mathrm{I})$ and the Effect of Finasteride Pretreatment in Cultured DFs of Human Hair Scalp

(A) The procollagen $\alpha(\mathrm{I})$ transcription level increased in a T dose dependent manner. The data in graph show are the densitometric quantification from six experiments and presented as mean \pm standard deviation (Student $t$-test, $* p<0.05 v s$. vehicle control). (B) Cultured human scalp DFs were treated with T and then culture supernatants were collected on $24 \mathrm{~h}$ for Western blot. T increased the expression level of type I procollagen protein. The data in graph are from six experiments and presented as mean \pm standard deviation (Student $t$-test, $* p<0.05$ vs. vehicle control). (C) The $\alpha 1$ (I) Procollagen transcription level increased by treatment of T (10 $\left.{ }^{-8} \mathrm{M}\right)$ and finasteride (10 $\left.{ }^{-7} \mathrm{M}\right)$. Procollagen $\alpha 1(\mathrm{I})$ transcription levels significantly decreased in pretreatment finasteride $\left(10^{-7} \mathrm{M}\right)$ before $\mathrm{T}\left(10^{-8} \mathrm{M}\right)(\mathrm{FT})$, compared with that of hormone alone (T). Finasteride alone treatment also increased the procollagen $\alpha(\mathrm{I})$ mRNA levels (F). The data are from five experiments and presented as mean \pm standard deviation ( $\dagger p<0.05$ androgen treatment $v s$. finasteride cotreatment, Student $t$-test). (D) Cultured human scalp DFs were treated with $\mathrm{T}\left(10^{-8} \mathrm{M}\right)$ and $\mathrm{F}\left(10^{-7} \mathrm{M}\right)$ and then culture supernatants were collected after $24 \mathrm{~h}$. T increased the expression level of type I procollagen protein. Finasteride also slightly increased because the role as synthetic 4-aza steroid compounds. The data is from five experiments with error bars representing the standard deviation ( $* p<0.05 v s$. vehicle control, $* * p<0.01 v s$. vehicle control, $\uparrow p<0.05$ androgen treatment $v s$. finasteride cotreatment, Student $t$-test).
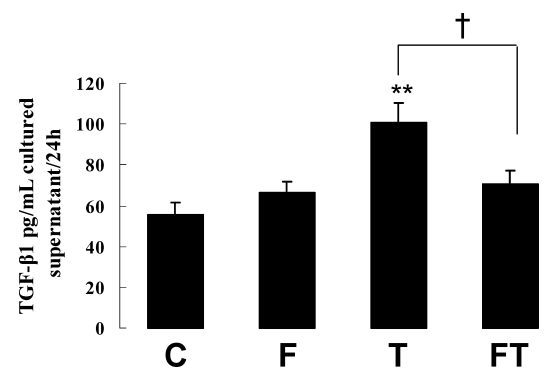

Fig. 2. ELISA Assessing the Expression Levels of TGF- $\beta 1$ in Scalp DFs

Cultured human scalp DFs were treated with $\mathrm{T}$ in the presence or absence of finasteride. $\mathrm{F}$; finasteride $\left(10^{-7} \mathrm{M}\right)$, $\mathrm{T}$; testosterone $\left(10^{-8} \mathrm{M}\right)$. Expression of TGF- $\beta 1$ is significantly lower in DFs pretreated finasteride than those with $\mathrm{T}$ treatment $(\dagger p<0.05)$. Graph is a presentation of three different experiments carried out in triplicate each time. Asterisks denotes $p<0.01$ compared with vehicle control, Student $t$-test.

forts to explain the pathogenetic mechanism of AGA had been made in hair epithelial cells as well as DPC. . $^{17,18,21-23)}$ Previous reports showed that the DHT and T treatment elicited an increase in the steady state concentration of $\alpha 1(\mathrm{I})$-procollagen mRNA in human osteoblast-like osteosarcoma cells ${ }^{24)}$ and that collagen synthesis was stimulated by

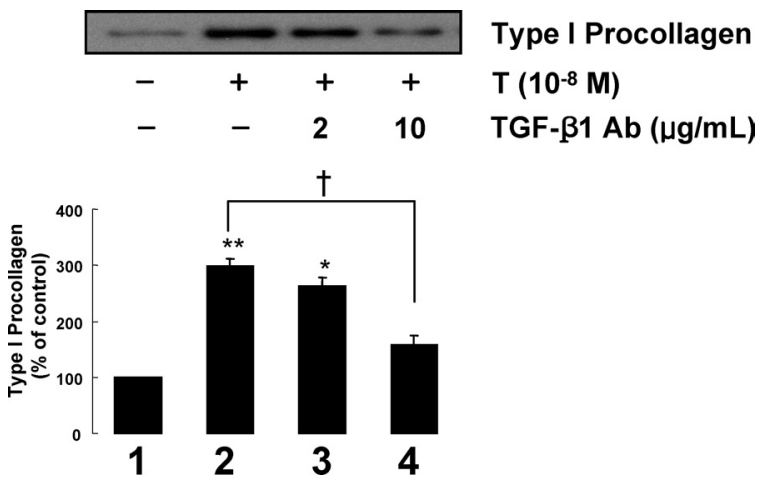

Fig. 3. Expression Level of the Type I Procollagen Is Inhibited by the Presence of a TGF- $\beta 1$ Neutralizing Ab in Scalp DFs

DFs were exposed to $\mathrm{T}\left(10^{-8} \mathrm{M}\right)$ in the presence or absence of a neutralizing $\mathrm{Ab}$ to TGF- $\beta 1(2,10 \mu \mathrm{g} / \mathrm{ml})$. Procollagen expression level was identified by Western blot. Cultured human DFs were treated with T $\left(10^{-8} \mathrm{M}\right)$ and TGF- $\beta 1(2,10 \mu \mathrm{g} / \mathrm{ml})$. Densitometric values for procollagen in the culture supernatants were determined. There is a significant increase in densitometric values for the procollagen in the supernatants in Texposed cells. This is blocked by the presence of the neutralizing TGF- $\beta 1 \mathrm{Ab}$. The data is from three independent experiments with error bars representing the standard deviation $(* p<0.05 v s$. vehicle control, $* * p<0.01 v s$. vehicle control, $\uparrow p<0.05$ androgen treatment $v s$. neutralizing TGF- $\beta 1 \mathrm{Ab}$ cotreatment, Student $t$-test). 
androgen in mice. ${ }^{25)}$ For that reason, perifollicular fibrosis may be another pathogenetic mechanisms of AGA, not a simple phenomenon usually seen in AGA.

This study clearly demonstrated that $\mathrm{T}$ stimulated the transcription and protein expression of type I procollagen gene in follicular DFs much more than vehicle-treated controls. This suggests that type I procollagen synthesis induction by androgen (T) may be, in part, responsible for densely packed collagen bundles around miniaturized hair follicles leading to perifollicular fibrosis seen in AGA. Finasteride treatment can also alleviate androgen-induced transcription and protein expression of type I procollagen in the follicular DFs. When a hair follicle miniaturizes, it ascends upward from the reticular dermis to the papillary dermis, followed by an associated angiofibrotic tract called a follicular streamer. ${ }^{26)}$ Considering above results and other report, the miniaturized hair can travel back down the streamer tract to the reticular dermis to resume its position and role as a terminal hair with finasteride treatment. These results ascertained as a basic mechanisms of finasteride to improve AGA in the aspect of DFs.

The type II $5 \alpha-\mathrm{R}$ is crucially involved in the pathogenesis of androgen-dependent hair growth. According to previous study, both DPC and DFs from occipital scalp hair expressed type I and II $5 \alpha$-R cDNA. ${ }^{11)}$ The type II gene was transcribed more in DFs than in DPC. ${ }^{11)}$ Finasteride is well known as a potent and highly selective $5 \alpha$-R type-II inhibitor. ${ }^{12,13)}$ It is reasonable that the action mechanisms of finasteride on AGA can be explained as inhibition of collagen synthesis in the follicular DFs, which lessens perifollicular fibrosis and formation of angiofibrotic tract involved in catagen induction.

TGF- $\beta 1$ appears to inhibit the rate of hair follicle lengthening in vitro and in vivo in mice to promote the regression phase of the organ culture of human hair follicles. ${ }^{8)}$ Thus, TGF- $\beta 1$ is considered as a catagen induction marker in hair follicle. ${ }^{9)}$ TGF- $\beta 1$ was shown to induce a rapid fibrotic response in vivo. ${ }^{27,28)}$ Moreover, chronic expression of TGF- $\beta 1$ in adult DFs caused severe alopecia characterized by epidermal and follicular hyperproliferation, apoptosis, as well as dermal fibrosis and inflammation. ${ }^{29)}$ Inui et al. ${ }^{23)}$ demonstrated that androgen induced TGF- $\beta 1$ from the balding dermal papilla cells plays an important role in miniaturization of hair shaft. Also, a key function for TGF- $\beta$ in wound healing and fibrosis is to regulate the expression of proteins of the fibrillar collagens and fibronectin. TGF- $\beta$ is considered a potent anabolic factor that enhances connective tissue deposition and repair and which sustained signaling likely leads to the development of tissue fibrosis. ${ }^{30)}$ Therefore, it can be inferred that the T-induced TGF- $\beta 1$ expression may induce perifollicular fibrosis in the DFs, accelerate hair follicles into moving on catagen phase of hair cycle, and inhibit epithelial growth, which brings all together out miniaturization of hair follicles in AGA. According to pretreatment of neutralizing TGF- $\beta 1$ antibody, T-induced type I procollagen level may decreased through the anti-TGF- $\beta 1$ protein in the DFs. This result was related to TGF- $\beta 1$, which induce catagen period fibrosis as the epitome of T-induced AGA. In this study, the finasteride and $\mathrm{T}$ co-treatment was demonstrated to reduce the expression of TGF- $\beta 1$ in scalp follicular DFs. This result also explains another possible action mechanisms of finasteride on AGA.

It is very difficult to obtain in vivo human scalp follicular cells. The scalp biopsies at the vertex and occiput in each person are much more difficult because of few volunteering. For that reason, the main limitation of this study is that it can not compare the results in vertex and occipital scalp follicular DFs. Although this study could not be performed under the comparative design, it still has valuable meanings that the vertex scalp follicular DFs have the active receptors response to androgen hormones and androgen hormone has effects on production of the collagen from follicular DFs, which shows histopathologically characteristic perifollicular fibrosis in AGA. In conclusion, perifollicular fibrosis induced by androgen can be suggested as one of the underlying pathogenetic mechanisms, resulting in hair follicle miniaturization in AGA. The biological characteristics of finasteride may be also explained as reducing T-induced procollagen and TGF$\beta 1$ synthesis, diminishing perifollicular fibrosis in AGA.

Acknowledgements This study was supported by a grant of the Korea Heath 21 R\&D Project, Ministry of Heath \& Welfare, Republic of Korea (03-PJ1-PG1-CH13-0001).

\section{REFERENCES}

1) Bergfeld W. F., Am. J. Med., 98, 95S-98S (1995).

2) Farthing M. J., Mattei A. M., Edwards C. R., Dawson A. M., Br. J. Dermatol., 107, 559-564 (1982).

3) Harris G., Azzolina B., Baginsky W., Cimis G., Rasmusson G. H., Tolman R. L., Raetz C. R., Ellsworth K., Proc. Natl. Acad. Sci. U.S.A., 89 10787-10791 (1992).

4) Jenkins E. P., Hsieh C. L., Milatovich A., Normington K., Berman D. M., Francke U., Russell D. W., Genomics, 11, 1102-1112 (1991).

5) Jaworsky C., Kligman A. M., Murphy G. F., Br. J. Dermatol., 127, 239-246 (1992)

6) Whiting D. A., J. Am. Acad. Dermatol., 28, 755-763 (1993).

7) Lattanand A., Johnson W. C., J. Cutan. Pathol., 2, 58-70 (1975).

8) Philpott M. P., Green M. R., Kealey T., J. Cell Sci., 97, 463-471 (1990).

9) Welker P., Foitzik K., Bulfone-Paus S., Henz B. M., Paus R., Arch. Dermatol. Res., 289, 554-557 (1997).

10) Kaufman K. D., Dermatol. Clin., 14, 697-711 (1996).

11) Lachgar S., Charveron M., Sarraute J., Mourard M., Gall Y., Bonafe J. L., J. Investig. Dermatol. Symp. Proc., 4, 290-295 (1999).

12) Olsen E., Aust. J. Dermatol., 38, A316 (1997).

13) Drake L., Hordinsky M., Fiedler V., Swinehart J., Unger W. P., Cotterill P. C., Thiboutot D. M., Lowe N., Jacobson C., Whiting D., Stieglitz S., Kraus S. J., Griffin E. I., Weiss D., Carrington P., Gencheff C., Cole G. W., Pariser D. M., Epstein E. S., Tanaka W., Dallob A., Vandormael K., Geissler L., Waldstreicher J., J. Am. Acad. Dermatol., 41, 550 554 (1999).

14) Hibberts N. A., Howell A. E., Randall V. A., J. Endocrinol., 156, 59 65 (1998)

15) Choudhry R., Hodgins M. B., Van der Kwast T. H., Brinkmann A. O., Boersma W. J., J. Endocrinol., 133, 467-475 (1992).

16) Liang T., Hoyer S., Yu R., Soltani K., Lorincz A. L., Hiipakka R. A., Liao S., J. Invest. Dermatol., 100, 663-666 (1993).

17) Rebora A., J. Am. Acad. Dermatol., 50, 777-779 (2004).

18) Itami S., Kurata S., Sonoda T., Takayasu S., Ann. N.Y. Acad. Sci., 642, 385-395 (1991).

19) Hamada K., Thornton M. J., Laing I., Messenger A. G., Randall V. A., J. Invest. Dermatol., 106, 1017-1022 (1996).

20) Thornton M. J., Hamada K., Messenger A. G., Randall V. A., J. Invest. Dermatol., 111, 727-732 (1998).

21) Pan H. J., Uno H., Inui S., Fulmer N. O., Chang C., Endocrine., 11, 321-327 (1999).

22) Bamberg E., Aichinger A., Mitteregger G., Vet. Dermatol., 15, 19-24 (2004).

23) Inui S., Fukuzato Y., Nakajima T., Yoshikawa K., Itami S., Faseb. J., 16, 1967-1969 (2002). 
24) Benz D. J., Haussler M. R., Thomas M. A., Speelman B., Komm B. S., Endocrinology, 128, 2723-2730 (1991).

25) Markova M. S., Zeskand J., McEntee B., Rothstein J., Jimenez S. A., Siracusa L. D., J. Invest. Dermatol., 123, 1052-1056 (2004).

26) Kligman A. M., Clin. Dermatol., 6, 108-118 (1998).

27) Roberts A. B., Sporn M. B., Assoian R. K., Smith J. M., Roche N. S., Wakefield L. M., Heine U. I., Liotta L. A., Falanga V., Kehrl J. H., An- thony S. F., Proc. Natl. Acad. Sci. U.S.A., 83, 4167-4171 (1986).

28) Sporn M. R., Shull J. H., Smith J. M., Ward J. M., Science, 219, 1329-1331 (1983).

29) Liu X., Alexander V., Vijayachandra K., Bhogte E., Diamond I., Glick A., Proc. Natl. Acad. Sci. U.S.A., 98, 9139-9144 (2001).

30) Mauviel A., Methods Mol. Med., 117, 69-80 (2005). 